\title{
The importance of an activity to improve disaster preparedness in the community through simulation of shelter management by residents
}

\author{
Sayaka SUMIDA ${ }^{1}$, Kaori MATSUO ${ }^{1}$, Akiko KUROTAKI ${ }^{2}$, Shihori KATSUNUMA ${ }^{3}$, \\ Marina INAGAKI ${ }^{1}$, Megumi ARISAKA ${ }^{1}$ and Aiko YAMAMOTO ${ }^{2}$ \\ ${ }^{1}$ University of Hyogo, Graduate School of Nursing Art and Science - Disaster Nursing Global Leader Degree Program, \\ Akashi, Hyogo, Japan \\ ${ }^{2}$ University of Hyogo - Research Institute of Nursing Care for People and Community, Akashi, Hyogo, Japan \\ ${ }^{3}$ University of Hyogo - Graduate School of Nursing Art Science, Akashi, Hyogo, Japan
}

This essay is a report of an activity that graduate students and faculties majoring in Disaster Nursing at the University of Hyogo conducted to allow local residents to improve their self-help and mutual-help skills for when disasters occur in the community. In Japan, gymnasiums and public facilities are used as temporary shelters when disasters occur. It is possible that residents have to live in a shelter for several days or even several months, depending on the type and scale of a disaster. Therefore, we believe that they need to experience living in a shelter in order to prepare for evacuation life. On May 17, 2015, the authors organized an activity that provided an opportunity for participants to imagine their lives in a shelter and also encouraged participants to think about how they would improve life in a shelter.

During this activity, a simulated shelter that participants could imagine and experience what life in the shelter would be like was made from cardboard. An information board, relief goods and an emergency kit, personal living space and shared spaces such as toilets and showers with models and photos were set inside his shelter. In addition, we showcased some problems that occurred during past disasters; they included a toilet that had poor sanitary condition (Fig. 1), relief supplies piled up, children who did not know what to do in their free time, an elderly person who had trouble walking to a restroom, a blind person who needed assistance, a person

Correspondence: Sayaka Sumida, University of Hyogo, 13-71 Kitaoji-cho, Akashi, Hyogo 673-8588, Japan. Email: jv.sumi.uz@ gmail.com

Received 9 February 2016; accepted 10 February 2016.

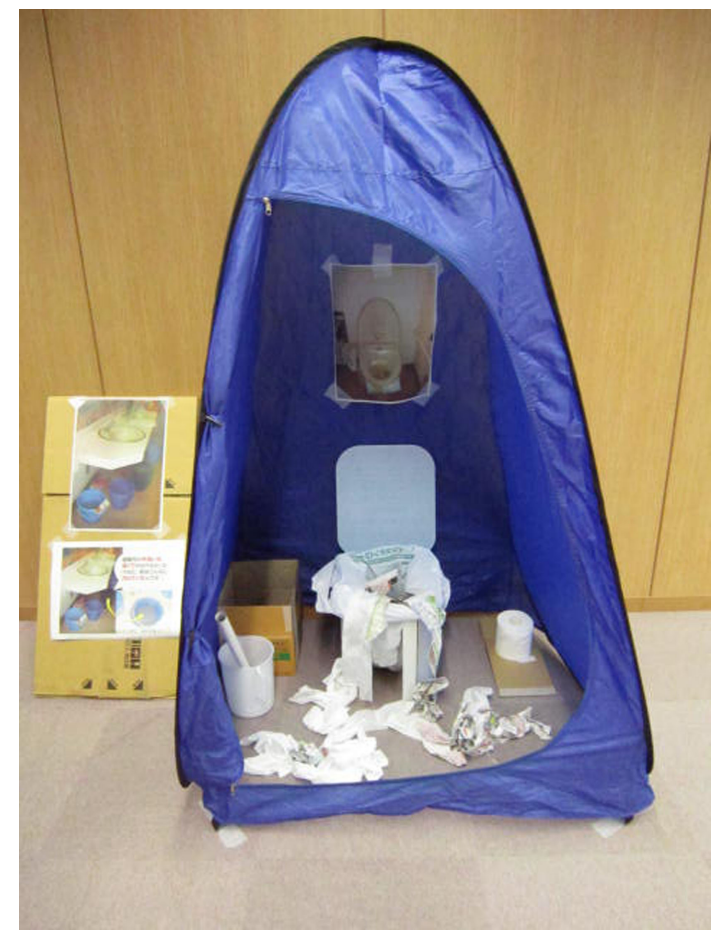

Figure 1 An example of a toilet within a shelter that has a poor sanitary condition.

who seemed sick, overflowing trash boxes, women who were looking for an area to breast-feed, posted crime information by police about a suspicious individual witness, and the temporary construction of showers with a long waiting line. Participants looked around this simulated shelter and they were asked, "What would you 


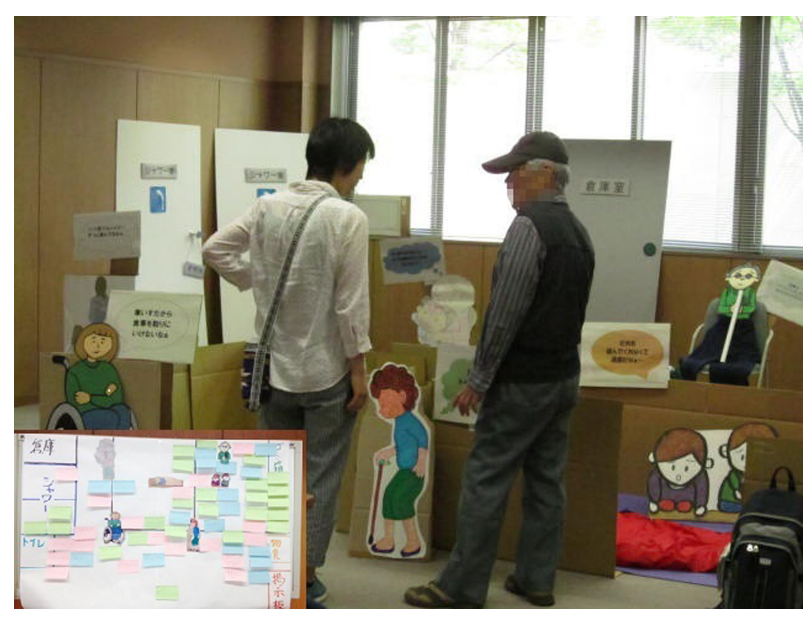

Figure 2 An example of a simulated shelter and a shelter map.

do if you lived in this shelter?". They wrote their responses on pieces of sticky paper, which were displayed on the shelter map showing the examples of problems (Fig. 2).

Approximately 70 people, not only Japanese but foreigners as well, from children to elderly people participated. Participants imagined their lives during the disaster and noticed some possible problems in the shelter; a total of 93 answers were obtained. For example, they could help by cleaning dirty toilets and overflowing trash boxes, make a queue to take turns using the showers, help to distribute relief supplies, make a free play space for children, play with children, and help vulnerable people to go to a restroom and to get food. There was also a suggestion about the necessity to secure a personal space based on the past evacuated experience of the participant. In contrast, there was no suggestion about crime prevention.

The following suggestions related to the consciousness of communal living, even though we did not highlight these as problems. Suggestions included trying to wash one's hands and wearing a mask for infection prevention, taking care of one's self so as not to be a nuisance to other people, and the necessity of areas for pets. Some elderly people said that they would not be able to do anything to contribute towards shelter management; however, they did say that they could take care of children in the shelter, after discussion with other participants and us. Moreover, they found that they could contribute positively; for instance, they were able to share their experience of previous disasters with younger people. Even to a person who did not seem to be interested, we were surprised to find that she was highly concerned and told us seriously about her own disaster preparedness. We found that this simulation was a good opportunity to raise awareness of disaster preparedness with participants. For example, some foreigners said that they did not know of any such activities in their own country and had no interest in disaster preparedness because of their inexperience of suffering from disasters. However, they were attracted by such activities to improve action in terms of disaster preparedness in Japan, and they obtained knowledge about disaster preparedness.

Children, elderly people, disabled people, foreigners, babies and pregnant women are defined as vulnerable populations during a disaster, and we set up some examples using such populations to show that they would need some assistance in such situations. However, people from these populations did inform us that they were able to find themselves various roles within a shelter in the event of a disaster. This activity made us think about what is necessary to help all evacuees have a role and take on responsibilities in a shelter without labelling such population as 'vulnerable people'.

This participatory activity attracted participants' interest and made them imagine what life in a shelter would be like. In contrast, some participants looked at a loss what to do in this activity because we did not explain contents to them at the beginning. Upon reflection, we should make a part of introduction to the simulation. In addition, the boundary between a part of introduction and a simulation is necessary in order to show participants into the simulation smoothly (e.g., drawing a distinct line using a curtain or a partition). In future, we would like to improve upon these simulation challenges and have these as part of promoting disaster preparedness.

Key words: shelter management, disaster preparedness, community, simulation 